\title{
Status of the Experimental Studies on DVMP and Transversity GPDs
}

\author{
Valery Kubarovsky* \\ and the CLAS collaboration \\ Thomas Jefferson National Accelerator Facility \\ Newport News, VA 23606, USA \\ E-mail: vpk@jlab.org
}

\begin{abstract}
The cross section of the exclusive $\pi^{0}$ and $\eta$ electroproduction reaction $e p \rightarrow e^{\prime} p^{\prime} \pi^{0} / \eta$ was measured at Jefferson Lab with a 5.75-GeV electron beam and the CLAS detector. Differential cross sections $d^{4} \sigma / d t d Q^{2} d x_{B} d \phi$ and structure functions $\sigma_{U}=\sigma_{T}+\varepsilon \sigma_{L}, \sigma_{T T}$ and $\sigma_{L T}$, as functions of $t$ were obtained over a wide range of $Q^{2}$ and $x_{B}$. At low $t$, both $\pi^{0}$ and $\eta$ are described reasonably well by Generalized Parton Distributions (GPDs) model in which chiral-odd transversity GPDs are dominant. Generalized form factors of the transversity GPDs $\left\langle H_{T}\right\rangle^{\pi, \eta}$ and $\left\langle\bar{E}_{T}\right\rangle^{\pi, \eta}$ were directly extracted from the experimental observables. The combined $\pi^{0}$ and $\eta$ data opens the way for the flavor decomposition of the transversity GPDs. The first ever demonstration of this decomposition was done for the transversity GPDs $H_{T}$ and $\bar{E}_{T}$. GPD $\bar{E}_{T}$ is connected with the density of the polarized quarks in an unpolarized nucleon in the impact parameter space. The spin density of polarized $u$ and $d$-quarks was evaluated for different values of Feynman $x$ from the GPD model tuned to described the experimental data.
\end{abstract}

23rd International Spin Physics Symposium - SPIN2018 -

10-14 September, 2018

Ferrara, Italy

* Speaker. 


\section{Introduction}

Understanding nucleon structure in terms of the fundamental degrees of freedom of Quantum Chromodynamics (QCD) is one of the main goals in the theory of strong interactions. In recent years it became clear that exclusive reactions may provide information about hadron structure encoded in so-called Generalized Parton Distributions [1,2] (GPDs). For each quark flavor $q$ there are eight GPDs. Four correspond to parton helicity-conserving (chiral-even) processes, denoted by $H^{q}, \tilde{H}^{q}, E^{q}$ and $\tilde{E}^{q}$, and four correspond to parton helicity-flip (chiral-odd) processes [3, 4], $H_{T}^{q}$, $\tilde{H}_{T}^{q}, E_{T}^{q}$ and $\tilde{E}_{T}^{q}$. The GPDs depend on three kinematic variables: $x, \xi$ and $t$. In a symmetric frame, $x$ is the average longitudinal momentum fraction of the struck parton before and after the hard interaction and $\xi$ (skewness) is half of the longitudinal momentum fraction transferred to the struck parton. The skewness can be expressed in terms of the Bjorken variable $x_{B}$ as $\xi \simeq x_{B} /\left(2-x_{B}\right)$. Here $x_{B}=Q^{2} /(2 p \cdot q)$ and $t=\left(p-p^{\prime}\right)^{2}$, where $p$ and $p^{\prime}$ are the initial and final four-momenta of the nucleon.

When the theoretical calculations for longitudinal virtual photons were compared with the JLab $\pi^{0}$ and $\eta$ data $[5,6,7]$ they were found to underestimate the measured cross sections by more than an order of magnitude in their accessible kinematic regions. The failure to describe the experimental results with quark helicity-conserving operators stimulated a consideration of the role of the chiral-odd quark helicity-flip processes. Deeply virtual meson electroproduction (DVMP), and in particular $\pi^{0}$ production in the reaction $e p \rightarrow e^{\prime} p^{\prime} \pi^{0}$, was identified $[8,9,10]$ as especially sensitive to the quark helicity-flip subprocesses. During the past few years, two parallel theoretical approaches - [8, 11] (GL) and [9, 10] (GK) have been developed utilizing the chiral-odd GPDs in the calculation of pseudoscalar meson electroproduction. The GL and GK approaches, though employing different models of GPDs, lead to transverse photon amplitudes that are much larger than the longitudinal amplitudes.

\section{Definition of structure functions}

The unpolarized reduced meson cross section is described by 4 structure functions $\sigma_{T}, \sigma_{L}$, $\sigma_{T T}$ and $\sigma_{L T}$ :

$$
2 \pi \frac{d^{2} \sigma\left(\gamma^{*} p \rightarrow p \pi^{0}\right)}{d t d \phi}=\frac{d \sigma_{T}}{d t}+\varepsilon \frac{d \sigma_{L}}{d t}+\varepsilon \frac{d \sigma_{T T}}{d t} \cos 2 \phi+\sqrt{2 \varepsilon(1+\varepsilon)} \frac{d \sigma_{L T}}{d t} \cos \phi .
$$

References $[10,11]$ obtain the following relations for unpolarized structure functions:

$$
\begin{gathered}
\frac{d \sigma_{L}}{d t}=\frac{4 \pi \alpha}{k^{\prime}} \frac{1}{Q^{4}}\left\{\left(1-\xi^{2}\right)|\langle\tilde{H}\rangle|^{2}-2 \xi^{2} \operatorname{Re}\left[\langle\tilde{H}\rangle^{*}\langle\tilde{E}\rangle\right]-\frac{t^{\prime}}{4 m^{2}} \xi^{2}|\langle\tilde{E}\rangle|^{2}\right\} \\
\frac{d \sigma_{T}}{d t}=\frac{4 \pi \alpha}{2 k^{\prime} Q^{4}}\left[\left(1-\xi^{2}\right)\left|\left\langle H_{T}\right\rangle\right|^{2}-\frac{t^{\prime}}{8 m^{2}}\left|\left\langle\bar{E}_{T}\right\rangle\right|^{2}\right] \\
\frac{d \sigma_{L T}}{d t}=\frac{4 \pi \alpha}{\sqrt{2} k^{\prime} Q^{4}} \xi \sqrt{1-\xi^{2}} \frac{\sqrt{-t^{\prime}}}{2 m} \operatorname{Re}\left[\left\langle H_{T}\right\rangle^{*}\langle\tilde{E}\rangle\right] \\
\frac{d \sigma_{T T}}{d t}=\frac{4 \pi \alpha}{k^{\prime} Q^{4}} \frac{t^{\prime}}{16 m^{2}}\left|\left\langle\bar{E}_{T}\right\rangle\right|^{2}
\end{gathered}
$$



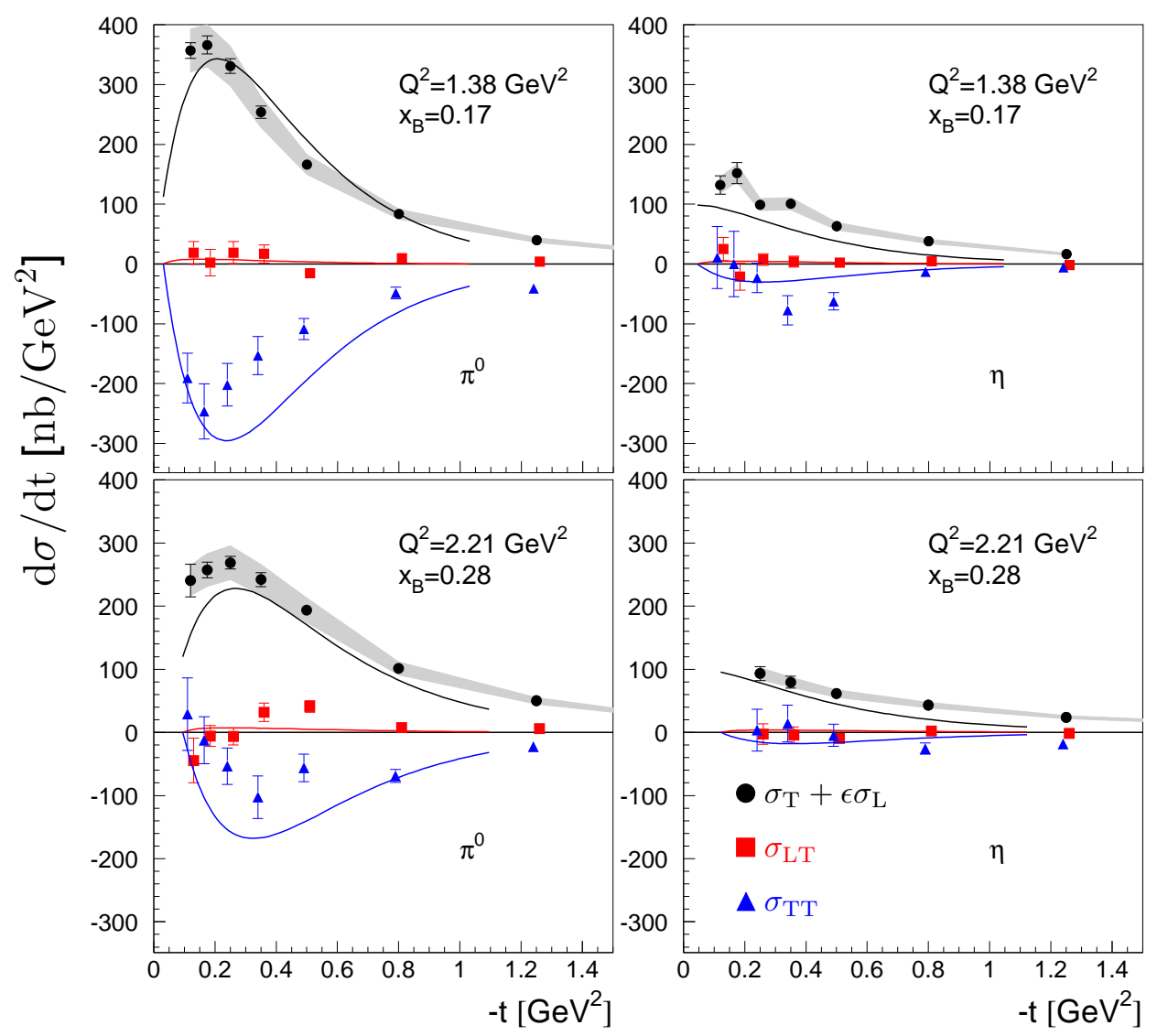

Figure 1: (Color online) The extracted structure functions vs. $t$ for the $\pi^{0}$ (left column) and $\eta$ (right column). The top row presents data for the kinematic point $\left(Q^{2}=1.38 \mathrm{GeV}^{2}, x_{B}=0.17\right)$ and bottom row for the kinematic point $\left(Q^{2}=2.21 \mathrm{GeV}^{2}, x_{B}=0.28\right)$. The data and curves are as follows: black circles $d \sigma_{U} / d t=d \sigma_{T} / d t+\varepsilon d \sigma_{L} / d t$, blue triangles - $d \sigma_{T T} / d t$, red squares - $d \sigma_{L T} / d t$. The error bars are statistical only. The gray bands are our estimates of the absolute normalization systematic uncertainties on $d \sigma_{U} / d t$. The curves are theoretical predictions produced with the GPG model of Goloskokov and Kroll [10].

Here $m$ is the mass of the proton, $t^{\prime}=t-t_{\min }$, where $\left|t_{\text {min }}\right|$ is the minimum value of $|t|$ corresponding to $\theta_{\pi}=0, k^{\prime}\left(Q^{2}, x_{B}\right)$ is a phase space factor and $\bar{E}_{T}=2 \widetilde{H}_{T}+E_{T}$. The brackets $\left\langle H_{T}\right\rangle$ and $\left\langle\bar{E}_{T}\right\rangle$ denote the convolution of the elementary process $\gamma^{*} q \rightarrow q \pi^{0}$ with the GPDs $H_{T}$ and $\bar{E}_{T}$. They are called them generalized form factors.

\section{Experimental data}

The cross section of the reaction $e p \rightarrow e p\left(\pi^{0} / \eta\right)$ measured by the CLAS collaboration at Jlab in bins of $Q^{2}, x_{B}, t$ and $\phi$ were published in Refs. [5, 6, 7]. Structure functions $\sigma_{U}=\sigma_{T}+\varepsilon \sigma_{L}, \sigma_{L T}$ and $\sigma_{T T}$ have been extracted from the angular distributions. These functions were compared with the predictions of the GPD models [10, 11]. The result confirmed that the measured unseparated cross sections are much larger than expected from leading-twist handbag calculations which are dominated by longitudinal photons. As an example, the comparison of the $\pi^{0}$ and $\eta$ structure 

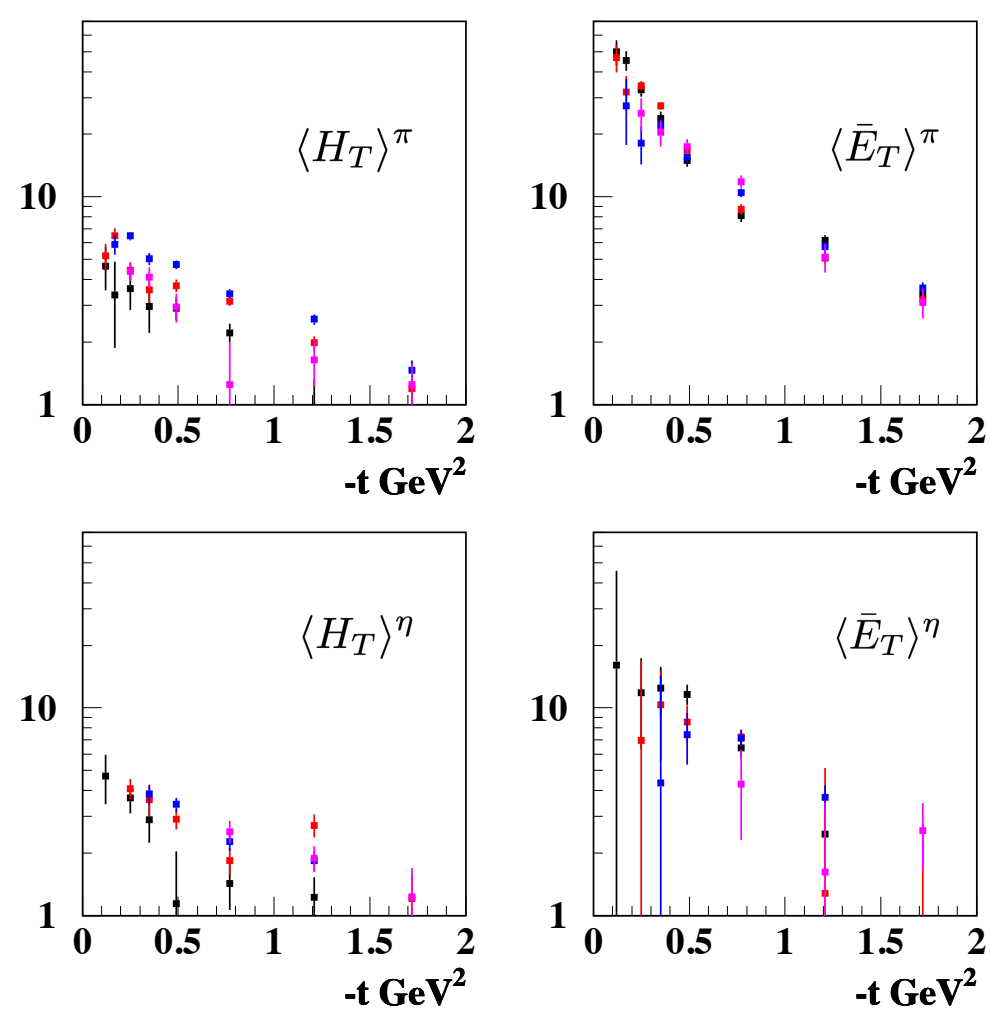

Figure 2: (Color online) Data points: CLAS. Top left: $\left|\left\langle H_{T}\right\rangle^{\pi}\right|$, top right: $\left|\left\langle\bar{E}_{T}\right\rangle^{\pi}\right|$, bottom left: $\left|\left\langle H_{T}\right\rangle^{\eta}\right|$, bottom right: $\left|\left\langle\bar{E}_{T}\right\rangle^{\eta}\right|$ as a function of $-t$ for different kinematics: $\left(Q^{2}=1.2 \mathrm{GeV}^{2}, x_{B}=0.15\right)$ black, $\left(Q^{2}=1.8\right.$ $\left.\mathrm{GeV}^{2}, x_{B}=0.22\right)$ red, $\left(Q^{2}=2.2 \mathrm{GeV}^{2}, x_{B}=0.29\right)$ blue, $\left(Q^{2}=2.7 \mathrm{GeV}^{2}, x_{B}=0.34\right)$ magenta.

functions is shown in Fig. 1 for two kinematical bins in $x_{B}$ and $Q^{2}$. The structure functions $\sigma_{U}$ and $\sigma_{T T}$ for $\eta$ are, respectively, factors of 2.5 and 10 smaller than for $\pi^{0}$. However, the GK GPD model [10] (curves) follows the experimental data. Taken together, the $\pi^{0}$ and $\eta$ results stringthen the statement about the transversity GPD dominance in the pseudoscalar electroproduction process.

\section{Generalized form factors}

The squared magnitudes of the generalized form factors $\left|\left\langle H_{T}\right\rangle\right|^{2}$ and $\left|\left\langle\bar{E}_{T}\right\rangle\right|^{2}$ may be directly extracted from the experimental data (see Eqs. 2.3 and 2.5) in the framework of GPD models.

$$
\begin{gathered}
\left|\left\langle\bar{E}_{T}\right\rangle^{\pi, \eta}\right|^{2}=\frac{k^{\prime} Q^{4}}{4 \pi \alpha} \frac{16 m^{2}}{t^{\prime}} \frac{d \sigma_{T T}^{\pi, \eta}}{d t} \\
\left|\left\langle H_{T}\right\rangle^{\pi, \eta}\right|^{2}=\frac{2 k^{\prime} Q^{4}}{4 \pi \alpha} \frac{1}{1-\xi^{2}}\left[\frac{d \sigma_{T}^{\pi, \eta}}{d t}+\frac{d \sigma_{T T}^{\pi, \eta}}{d t}\right] .
\end{gathered}
$$

Figure 2 presents the modulus of the generalized form factors $\left|\left\langle H_{T}\right\rangle^{\pi}\right|,\left|\left\langle\bar{E}_{T}\right\rangle^{\pi}\right|,\left|\left\langle H_{T}\right\rangle^{\eta}\right|$ and $\left|\left\langle\bar{E}_{T}\right\rangle^{\eta}\right|$ for 4 different kinematics. Note the dominance of the $\left|\left\langle\bar{E}_{T}\right\rangle\right|$ over $\left|\left\langle H_{T}\right\rangle\right|$ for both $\pi^{0}$ and $\eta$. Generalized form factors $\left\langle H_{T}\right\rangle^{\pi}$ and $\left\langle\bar{E}_{T}\right\rangle^{\pi}$ are shown in more detail in Fig. 3. The $\left\langle\bar{E}_{T}\right\rangle^{\pi}$ formfactor 
has steeper t-dependemce than $\left\langle H_{T}\right\rangle^{\pi}$. The t-slope parameters, obtained by an exponential fit of the form $e^{b t}$, are $b\left(\left\langle\bar{E}_{T}\right\rangle\right)=1.27 \mathrm{GeV}^{-2}$ and $b\left(\left\langle H_{T}\right\rangle\right)=0.98 \mathrm{GeV}^{-2}$.

\section{Flavor decomposition}

In electroproduction of $\pi^{0}$ and $\eta$ mesons the GPDs $F_{i}$ appears in the following combinations

$$
\begin{gathered}
F_{i}^{\pi}=\frac{1}{\sqrt{2}}\left[e_{u} F_{i}^{u}-e_{d} F_{i}^{d}\right] \\
F_{i}^{\eta}=\frac{1}{\sqrt{6}}\left[e_{u} F_{i}^{u}+e_{d} F_{i}^{d}-2 e_{s} F_{i}^{s}\right]
\end{gathered}
$$

The $q$ and $\bar{q}$ GPDs contribute in the quark combinations $F_{i}^{q}-F_{i}^{\bar{q}}$. Hence there is no contribution from the strange quarks if we assume that $F_{i}^{s} \simeq F_{i}^{\bar{s}}$. For flavor decomposition we have to take into account the decay constants $f_{\pi}$ and $f_{\eta}$, the chiral condensate constants $\mu_{\pi^{0}}=2.57 \mathrm{GeV}$, $\mu_{1}=0.958 \mathrm{GeV}$ and $\mu_{8}=2.32 \mathrm{GeV}$, and the contribution from singlet and octet $\eta$ states [10].

$$
F_{i}^{\eta}=F_{i}^{\pi}\left(\cos \theta_{8}-\sqrt{2} \frac{\mu_{1}}{\mu_{8}} \frac{f_{1}}{f_{8}} \sin \theta_{1}\right) \frac{f_{8}}{f_{\pi^{0}}} \frac{\mu_{8}}{\mu_{\pi^{0}}}=\frac{F_{i}^{8}}{k_{\eta}},
$$

where the mixing angles are: $\theta_{8}=-21.2^{\circ}$ and $\theta_{1}=-9.2^{\circ}$. The octet and singlet wave functions are very similar and the decay constants are close as well $f_{8}=1.26 f_{\pi}$ and $f_{1}=1.17 f_{\pi}$. The overall factor for the $\eta$ meson is $k_{\eta}=0.863$. Using $e_{u}=\frac{2}{3}$ and $e_{d}=-\frac{1}{3}$ we will end up with

$$
\begin{aligned}
F_{i}^{\pi} & =\frac{1}{3 \sqrt{2}}\left[2 F_{i}^{u}+F_{i}^{d}\right] \\
k_{\eta} F_{i}^{\eta} & =\frac{1}{3 \sqrt{6}}\left[2 F_{i}^{u}-F_{i}^{d}\right] .
\end{aligned}
$$

Experimentally we have access only to the $\left|\left\langle F_{i}{ }^{\pi}\right\rangle\right|^{2}$ and $\left|\left\langle F_{i}^{\eta}\right\rangle\right|^{2}$ (see Eq. 4.1-4.2). The final equation for the $\left\langle H_{T}\right\rangle$ convolution reads

$$
\begin{gathered}
\frac{1}{18}\left|2\left\langle H_{T}\right\rangle^{u}+\left\langle H_{T}\right\rangle^{d}\right|^{2}=\left|\left\langle H_{T}\right\rangle^{\pi}\right|^{2} \\
\frac{1}{54}\left|2\left\langle H_{T}\right\rangle^{u}-\left\langle H_{T}\right\rangle^{d}\right|^{2}=k_{\eta}^{2}\left|\left\langle H_{T}\right\rangle^{\eta}\right|^{2} .
\end{gathered}
$$

and simular equations for $\left\langle\bar{E}_{T}\right\rangle$.

The solution of these equations will lead to the flavor decomposition of the generalized form factors $\left\langle H_{T}\right\rangle^{u}$ and $\left\langle H_{T}\right\rangle^{d}$ as well as $\left\langle\bar{E}_{T}\right\rangle^{u}$ and $\left\langle\bar{E}_{T}\right\rangle^{d}$. However, the convolution integrals have real and imaginary parts. So it is impossible to solve these equations unambiguously with only two equations in hands. So, in order to estimate the form factors, we make an ad hoc assumption that the relative phase $\Delta \phi$ between $\left\langle H_{T}\right\rangle^{u}$ and $\left\langle H_{T}\right\rangle^{d}$ equals 0 or 180 degrees. Ignoring an overall phase, the form factors are then real, and we arbitrarily choose the solution with $\left\langle H_{T}\right\rangle^{u}$ and $\left\langle\bar{E}_{T}\right\rangle^{u}$ positive. Fig. 4 presents $\left\langle H_{T}\right\rangle^{u},\left\langle H_{T}\right\rangle^{d},\left\langle\bar{E}_{T}\right\rangle^{u}$ and $\left\langle\bar{E}_{T}\right\rangle^{d}$ for one kinematic point $\left(Q^{2}=2.2 \mathrm{GeV}^{2}, x_{B}=0.27\right)$ calculated with this assumption. Note the different signs of $\left\langle H_{T}\right\rangle^{u}$ and $\left\langle H_{T}\right\rangle^{d}$ and the same sign of $\left\langle\bar{E}_{T}\right\rangle^{u}$ and $\left\langle\bar{E}_{T}\right\rangle^{d}$. The theoretical predictions of the large- $N_{c}$ QCD model [12] are consistent with this spin-flavor structure extracted from hard exclusive $\pi^{0}$ and $\eta$ electroproduction data. 


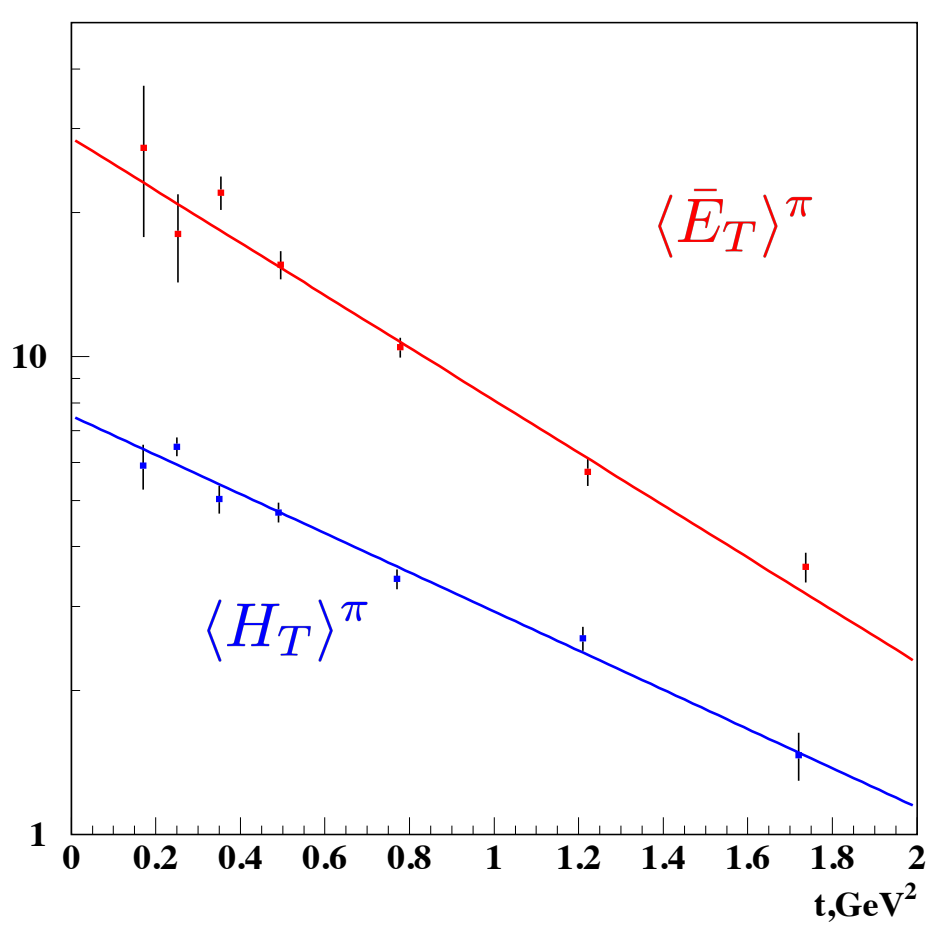

Figure 3: (Color online) Generalized form factors $\left|\left\langle H_{T}\right\rangle^{\pi}\right|$ and $\left|\left\langle\bar{E}_{T}\right\rangle^{\pi}\right|$ as a function of $-t$ for $Q^{2}=2.2 \mathrm{GeV}^{2}$ and $x_{B}=0.27$. Top: $\left|\left\langle\bar{E}_{T}\right\rangle^{\pi}\right|$ in red; Bottom: $\left|\left\langle H_{T}\right\rangle^{\pi}\right|$ in blue.

\section{Quark spin densities in the transverse plane and generalized transversity distributions}

Two-dimensional Fourier transforms of GPDs $H\left(x, \xi=0,-\vec{\Delta}^{2}\right)$ and $\bar{E}_{T}\left(x, 0,-\vec{\Delta}^{2}\right)$, where $\vec{\Delta}^{2}=-t$, define the spin density of the polarized quarks in an unpolarized proton [13].

$$
\begin{aligned}
H(x, \vec{b}) & =\int \frac{d^{2} \vec{\Delta}}{(2 \pi)^{2}} e^{-i \vec{b} \vec{\Delta}} H\left(x, 0,-\vec{\Delta}^{2}\right) \\
\bar{E}_{T}(x, \vec{b}) & =\int \frac{d^{2} \vec{\Delta}}{(2 \pi)^{2}} e^{-i \vec{b} \vec{\Delta}} \bar{E}_{T}\left(x, 0,-\vec{\Delta}^{2}\right)
\end{aligned}
$$

The GPDs $\bar{E}_{T}(x, \xi=0, t)$ and $H(x, \xi=0, t)$ were parametrized in the form [14]

$$
\bar{F}^{q}(x, \xi=0, t)=g^{q}(x) \cdot \exp \left[\left(f^{q}(x) t\right],\right.
$$

where $g^{q}(x)$ and $f^{q}(x)$ are the GPD forward limit and profile functions respectively. The Fourier transform for this parametrization reads

$$
\bar{F}^{q}(x, b)=\frac{1}{4 \pi} \frac{g^{q}(x)}{f^{q}(x)} \exp \left[\frac{-b^{2}}{4 f^{q}(x)}\right] .
$$

For quarks polarized along $b_{x}$-axis the impact parameter density reads [13]

$$
\delta^{q}(x, \vec{b})=\frac{1}{2}\left[H^{q}(x, \vec{b})-\frac{b_{y}}{m} \frac{\partial}{\partial b^{2}} \bar{E}_{T}^{q}(x, \vec{b})\right] .
$$



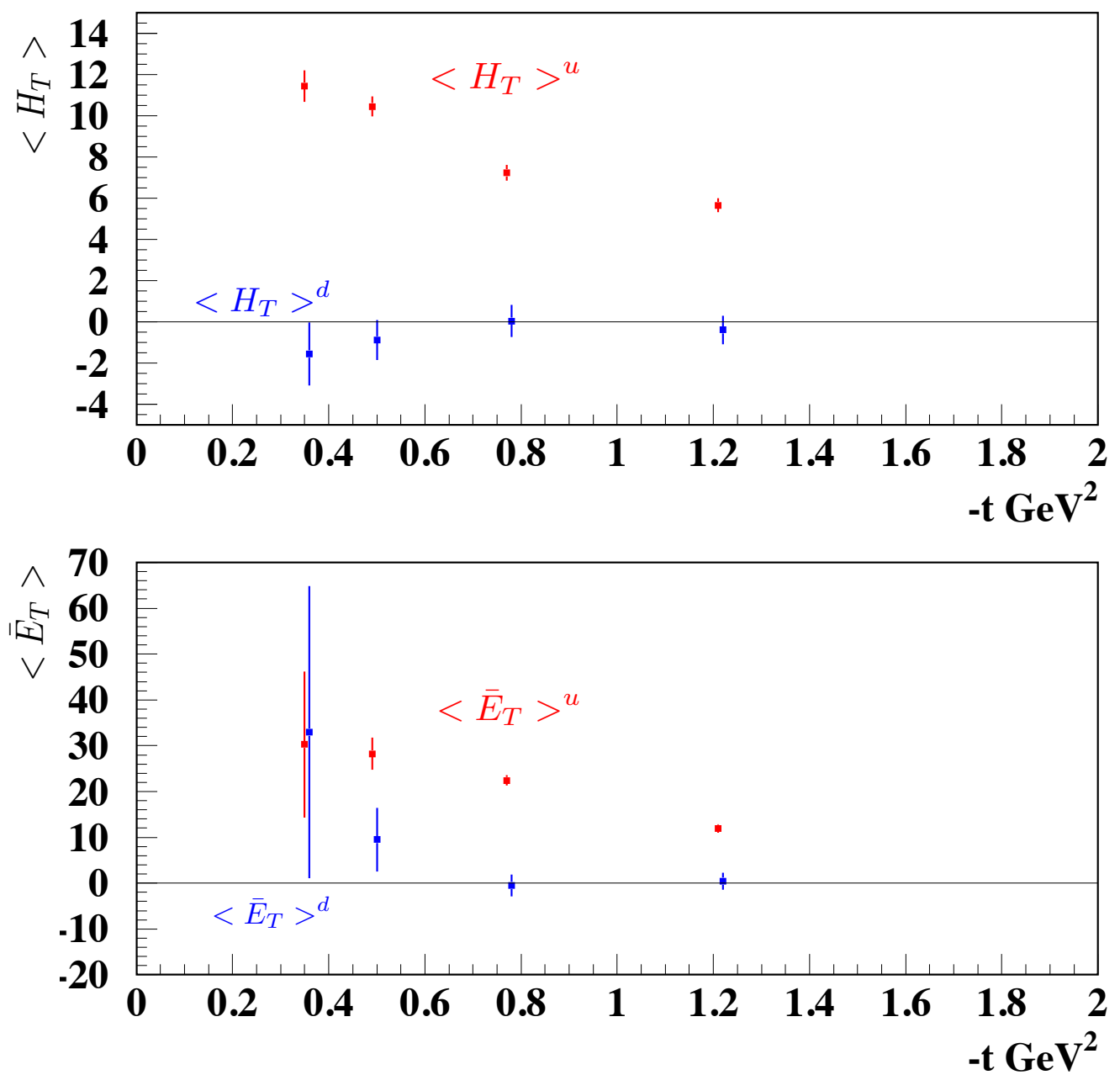

Figure 4: (Color online) Flavor separated generalized form factors $\left\langle H_{T}\right\rangle$ and $\left\langle\bar{E}_{T}\right\rangle$ as a function of - $t$ for $Q^{2}=2.2 \mathrm{GeV}^{2}$ and $x_{B}=0.27$. Top: $\left\langle H_{T}\right\rangle^{u}$ (red) and $\left\langle H_{T}\right\rangle^{d}$ (blue); Bottom: $\left\langle\bar{E}_{T}\right\rangle^{u}$ (red) and $\left\langle\bar{E}_{T}\right\rangle^{d}$ (blue).

The GPD $H(x, \vec{b})$ describes the density of unpolarized quarks and $\bar{E}_{T}(x, \vec{b})$ is related to the distortion of the polarized quark distribution in the transverse plane. We can map the $u$ and $d$-quark transverse spin density distributions as a function of Feynman $x$ based on the GPD model [10] tuned to describe the CLAS data. For example, Fig. 5 shows the impact parameter density of transversely polarized quarks along the $b_{x}$-axis in an unpolarized proton for Feynman $x=0.1$ and $x=0.2$. Note the distortion along $b_{y}$-axis, similar for $\mathrm{u}$ and d-quarks. Looking at the transverse quark density distribution, we can say that this width is diminished as $x \rightarrow 1$. This behavior is typical for the GPD models. 


\section{Polarized Quarks in Unpolarized Proton}
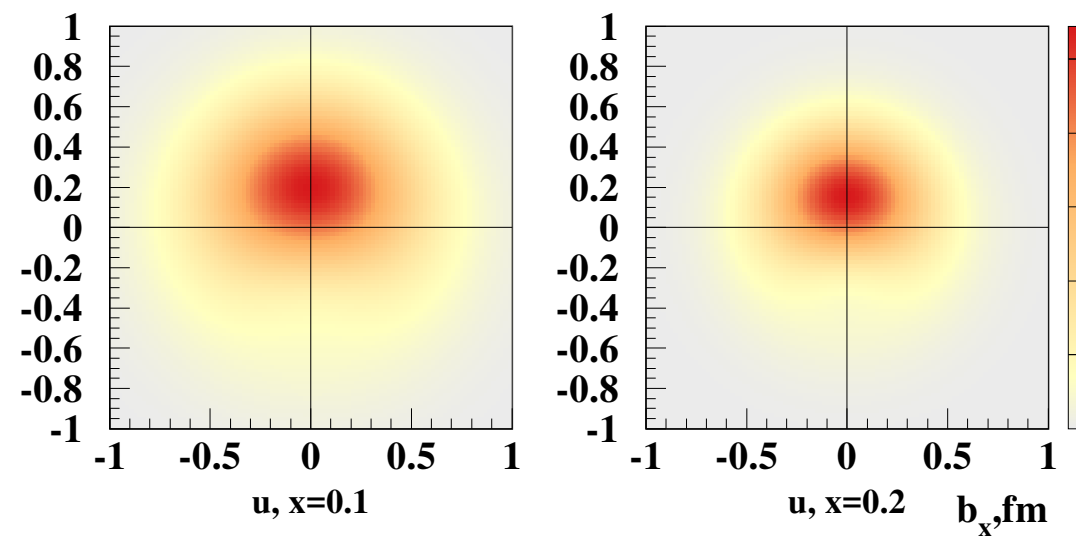

0.005

0.004

0.003

0.002

0.001
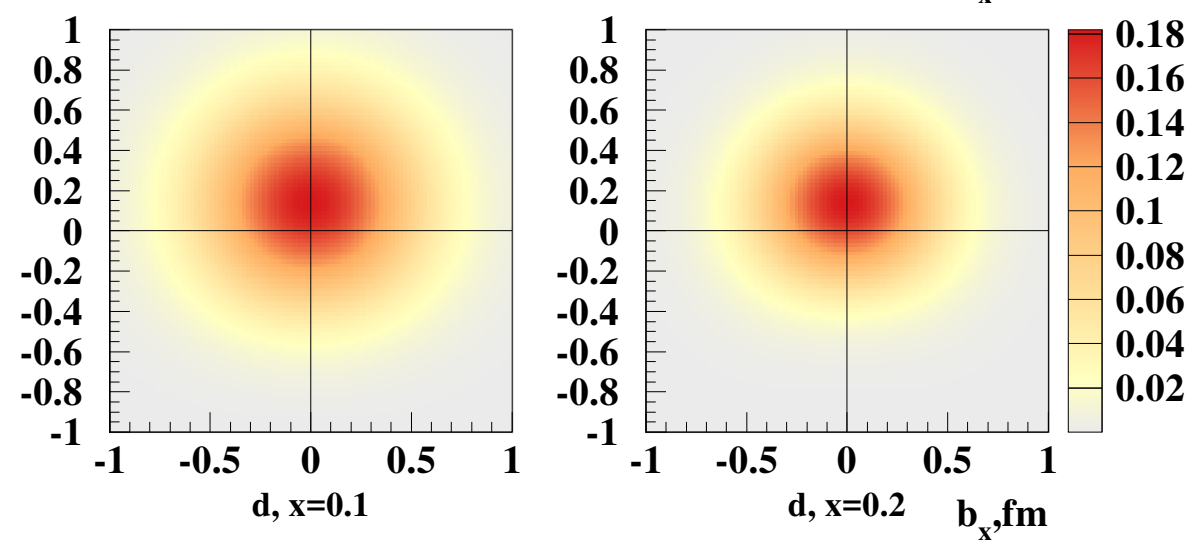

Figure 5: (Color online) Impact parameter density of quarks which are transversely polarized along $b_{x}$-axis in an unpolarized proton. Top left and right panels are for $\mathrm{u}$-quarks with $x=0.1$ and $x=0.2$, respectively. Bottton left and right panels are for d-quarks with $x=0.1$ and $x=0.2$.

\section{Conclusion}

Differential cross sections of exclusive $\pi^{0}$ and $\eta$ electroproduction have been obtained in the few-GeV region at more than 1800 kinematic points in bins of $Q^{2}, x_{B}, t$ and $\phi_{\pi}$. Virtual photon structure functions $\sigma_{U}, \sigma_{T T}$ and $d \sigma_{L T}$ have been obtained. It is found that $\sigma_{U}$ and $\sigma_{T T}$ are comparable in magnitude with each other, while $\sigma_{L T}$ is very much smaller than either. Generalized form factors of the transversity GPDs $\left\langle H_{T}\right\rangle^{\pi, \eta}$ and $\left\langle\bar{E}_{T}\right\rangle^{\pi, \eta}$ were directly extracted from the experimental observables for the first time. It was found that the GPD $\bar{E}_{T}$ dominates in pseudoscalar meson production. The combined $\pi^{0}$ and $\eta$ data opens the way for the flavor decomposition of the transversity GPDs. Within some simplifying assumptions, the decomposition has been demonstrated. The spin density of polarized $u$ and $d$-quarks in the transverse plane was evaluated for different values of $x$ from the GPD model tuned to described the experimental data.

\section{Acknowledgments}

The author thanks G. Goldstein, S. Goloskokov, P. Kroll, S. Liuti, A. Radyushkin, P. Schweitzer 
and C. Weiss for many informative discussions and making available the results of their calculations. This material is based upon work supported by the U.S. Department of Energy, Office of Science, Office of Nuclear Physics under contract DE-AC05-06OR23177.

\section{References}

[1] X. Ji, Phys. Rev. Lett. 78, 610 (1997); Phys. Rev. D 55, 7114 (1997).

[2] A.V. Radyushkin, Phys. Lett. B 380, 417 (1996); Phys. Rev. D 56, 5524 (1997).

[3] P. Hoodbhoy and X. Ji, Phys. Rev. D 58, 054006 (1998).

[4] M. Diehl, Phys. Rep. 388, 41 (2003) and references within.

[5] I. Bedlinskiy et al. (CLAS Collaboration), Phys. Rev. Lett. 109, 112001 (2012).

[6] I. Bedlinskiy et al. (CLAS Collaboration), Phys. Rev. C 90, 025205 (2014).

[7] I. Bedlinskiy et al. (CLAS Collaboration), Phys. Rev. C 95, 035202 (2017).

[8] S. Ahmad, G. R. Goldstein and S. Liuti, Phys. Rev. D 79, 054014 (2009).

[9] S. V. Goloskokov and P. Kroll, Eur. Phys. J. C 65, 137 (2010).

[10] S. V. Goloskokov and P. Kroll, Eur. Phys. J. A 47, 112 (2011).

[11] G. Goldstein, J. O. Gonzalez-Hernandez and S. Liuti, Phys. Rev. D 84, 034007 (2011); Int. J. Mod. Phys. Conf. Ser. 20, 222 (2012); J. Phys. G: Nucl. Part. Phys. 39115001 (2012).

[12] P. Schweitzer and C. Weiss, Phys. Rev. C 94, 045202 (2016).

[13] M. Diehl and Ph. Hagler, Eur. Phys. J. C44, 87 (2005).

[14] M. Diehl and P. Kroll, Eur. Phys. J. C73, 2397 (2013). 